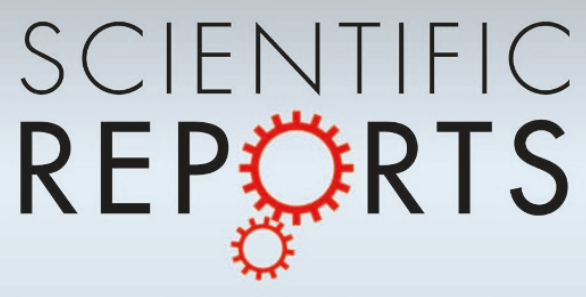

OPEN

SUBJECT AREAS:

VOLTAGE CLAMP

POTASSIUM CHANNELS

BIOLOGICAL FLUORESCENCE

FLUORESCENT PROTEINS

Received

25 September 2012

Accepted

6 December 2012

Published

8 January 2013

Correspondence and requests for materials should be addressed to B.C. (bchanda@wisc. edu)

\section{Function of Shaker potassium channels produced by cell-free translation upon injection into Xenopus oocytes}

\author{
Brian W. Jarecki', Shin-ichi Makino², Emily T. Beebe'2, Brian G. Fox² \& Baron Chanda'
}

'Department of Neuroscience, University of Wisconsin-Madison, Madison, Wl, 53706, ${ }^{2}$ Transmembrane Protein Center, Center for Eukaryotic Structural Genomics, Department of Biochemistry, University of Wisconsin-Madison, Madison, WI, 53706.

Voltage-gated ion channels are a class of membrane proteins that temporally orchestrate the ion flux critical for chemical and electrical signaling in excitable cells. Current methods to investigate the function of these channels rely on heterologous expression in living systems or reconstitution into artificial membranes; however these approaches have inherent drawbacks which limit potential biophysical applications. Here, we describe a new integrated approach combining cell-free translation of membrane proteins and in vivo expression using Xenopus laevis oocytes. In this method, proteoliposomes containing Shaker potassium channels are synthesized in vitro and injected into the oocytes, yielding functional preparations as shown by electrophysiological and fluorescence measurements within few hours. This strategy for studying eukaryotic ion channels is contrasted with existing, laborious procedures that require membrane protein extraction and reconstitution into synthetic lipid systems.

tudying the functional properties of ion channels requires an experimental setup that allows detection of ion flux across a membrane. Currently, heterologous expression in living hosts ${ }^{1}$ and reconstitution of purified proteins into artificial membranes ${ }^{2}$ provide the best options for biophysical research of ion channels. However, each method has inherent drawbacks, which limit their potential application. For example, while a variety of channels can be expressed in Xenopus laevis oocytes or cultured cells, subsequent labeling for spectroscopic studies is restricted to accessible extracellular sites. On the other hand, use of artificial membranes for ion channel studies allows one to examine purified proteins and label most sites prior to reconstitution of the protein. Nonetheless, this approach can be laborious and result in low protein yield. Therefore, when investigating the biological complexities associated with ion channels, it would be of significant value to have an alternative approach that combines the advantages and overcomes the limitations of these two methods.

Cell-free translation offers unique opportunities for synthesis of integral membrane proteins (IMPs) by circumventing the inherent biological variability associated with living expression hosts ${ }^{3}$. Using a wheat-germ cell-free translation system, our lab $b^{4,5}$ and others ${ }^{6,7}$ have produced polytopic IMPs in sufficient amounts for functional in vitro analysis. Moreover, cell-free approaches allow for inclusion of supplemental reagents and implementing selective labeling strategies during protein translation ${ }^{3}$.

Xenopus oocytes are widely used as an in vivo system to study ion channels upon injection with mRNA ${ }^{8}$, and more recently, as a biological system for in-cell $\mathrm{NMR}^{9}$. Miledi and colleagues first described a novel technique to 'microtransplant' functional channels into oocytes by injecting either isolated membrane vesicles ${ }^{10}$ or reconstituted proteoliposomes containing processed channels from the Torpedo electroplaque ${ }^{11}$. The group later extended the approach to transplant membrane channels from cultured human embryonic kidney (HEK293) cells $^{12}$, human muscle biopsies ${ }^{13}$, and brains ${ }^{14}$ to examine function and pharmacology. These studies primarily rely on efficient extraction of functional channels from a natural membrane to be either directly transplanted or incorporated, upon vesicular reconstitution, into the foreign oocyte membrane. Work from Schmidt and MacKinnon ${ }^{15}$ and Le Caherec et al. ${ }^{16}$ also demonstrated functional properties of two different membrane channels when the purified protein was reconstituted into lipid vesicles and injected into oocytes.

While fundamentally intriguing, the aforementioned strategies for channel incorporation into the oocyte membrane have notable limitations pertaining to sample preparation. For example, the ability to express, isolate and purify, or reconstitute the protein of interest is time-consuming and may not be suitable for proteins whose overexpression is toxic to the cells ${ }^{17}$. Furthermore, IMPs may lose their functionality during the purification process upon exposure to detergents. Here we describe a system wherein mRNA transcripts are used as a 'feed' for 
eukaryotic in vitro translation reactions containing a wheat-germ cell extract, liposomes, amino acids, and other reagents necessary to facilitate protein synthesis ${ }^{3}$. Upon synthesis of the membrane protein and concomitant insertion into liposomes, the proteoliposomes are concentrated by dialysis and centrifugation. During the entire procedure, which requires less than $24 \mathrm{~h}$, channels are not exposed to detergents and functionality is tested by directly injecting proteoliposomes into Xenopus laevis oocytes. We show that this strategy enables us to functionally incorporate labeled proteins into the oocyte membrane. In contrast to present cellular labeling techniques, our method provides a way to quantitatively estimate the extent of labeling for a target protein. Together, these findings demonstrate the feasibility of this approach for functional and spectroscopic studies of in vitro modified ion channels, which in this case is the exemplary ion channel-the Shaker potassium channel.

\section{Results}

Optimizing cell-free expression and incorporation for in vivo functional studies. We used multiple in vitro transcription and translation conditions to optimize the yield of the synthesized proteins for injection into oocytes (Fig. 1). For the initial trials, we used GFP-fusions (Methods) with a predicted molecular mass of 97 and $32 \mathrm{kDa}$ for the zh4IR monomer and cytb5 respectively (Fig. S1). Since the cell-free extract consists of soluble components, synthesized channels could be recovered in a purified state by resuspension of the pellet fraction. Inclusion of exogenous liposomes during translation facilitated channel precipitation. This single step purification yielded a proteoliposome suspension without use of detergents. SDS-PAGE analyses of non-fused zh4IR channel monomers (71 kDa) indicated that the samples were $\sim 60 \%$ pure and lacked heavy glycosylation ${ }^{18}$ and disulfide bonds (Fig. S2).
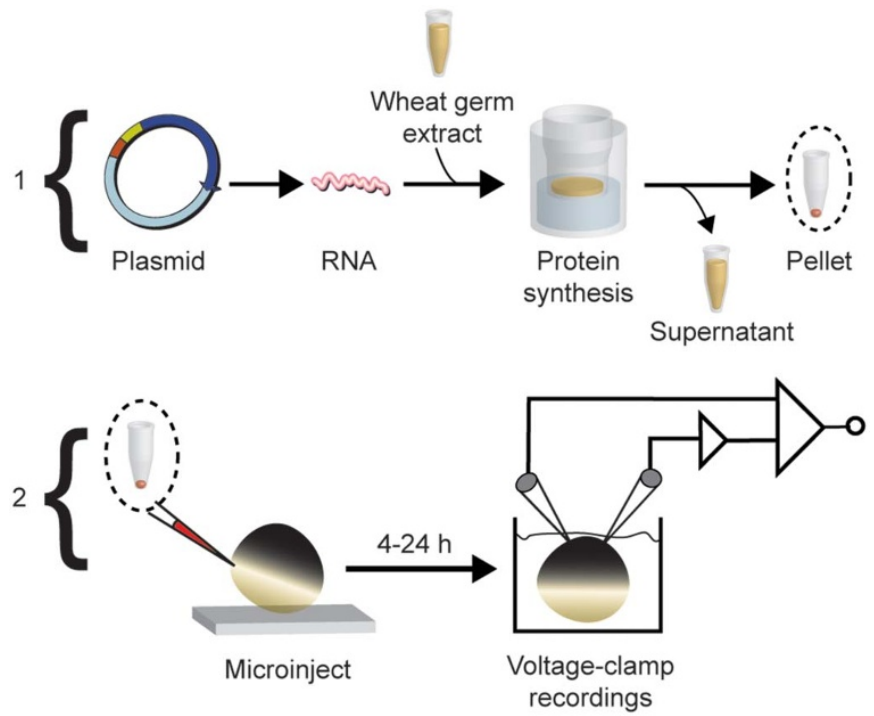

Figure 1 | Cartoon workflow diagram of cell-free protein production and expression in Xenopus laevis oocytes illustrated in two phases. Phase 1: Unique $5^{\prime}$ and $3^{\prime}$ restriction sites appended onto a target gene openreading frame are used to digest and subclone into a specialized plasmid (pEU-HSBC) for cell-free synthesis. The plasmid DNA is used as a template for in vitro transcription of RNA. Transcribed messenger RNA directs protein translation in wheat-germ cell-free extract supplemented with liposomes to make proteoliposomes. Since the Shaker channels can be recovered from the pellet fraction, the supernatant is discarded and the proteoliposomes are collected after centrifugation. Phase 2: Samples are reconstituted to specified concentrations in a salt buffer and injected into single Xenopus laevis oocytes at the vegetal equator. Currents are measured under voltage-clamp within $24 \mathrm{~h}$.
Quantified concentrations of reconstituted proteins were injected into oocytes (Fig. 2a). We tested the function of injected wildtype and mutant (M356C) channels using voltage-clamp electrophysiology. Additionally, the complete translation mixture and a purified non voltage-dependent membrane-anchored protein (GFP-cytb5t) were injected as controls and compared to uninjected oocytes. We hypothesized that if functional membrane bound channels were inserted in the correct 'inside-in' orientation, we would observe outward $\mathrm{K}^{+}$current in response to depolarizing voltage steps. Currents elicited from the Shaker potassium channel injections displayed a typical voltage-dependent response (Fig. $2 c-f$ ), similar to that of the mRNA (pBSTA vector) injected channels (Fig. S3). These results indicate that the channel function is not altered by proteoliposome formation or by subsequent insertion into the oocyte membrane ${ }^{19}$. The electrophysiological response for GFP-cytb5t was indistinguishable from uninjected oocytes (Fig. 2b). Interestingly, injection of the unpurified translation mixture containing the potassium channel was detrimental to the oocyte health (Methods) suggesting a simple
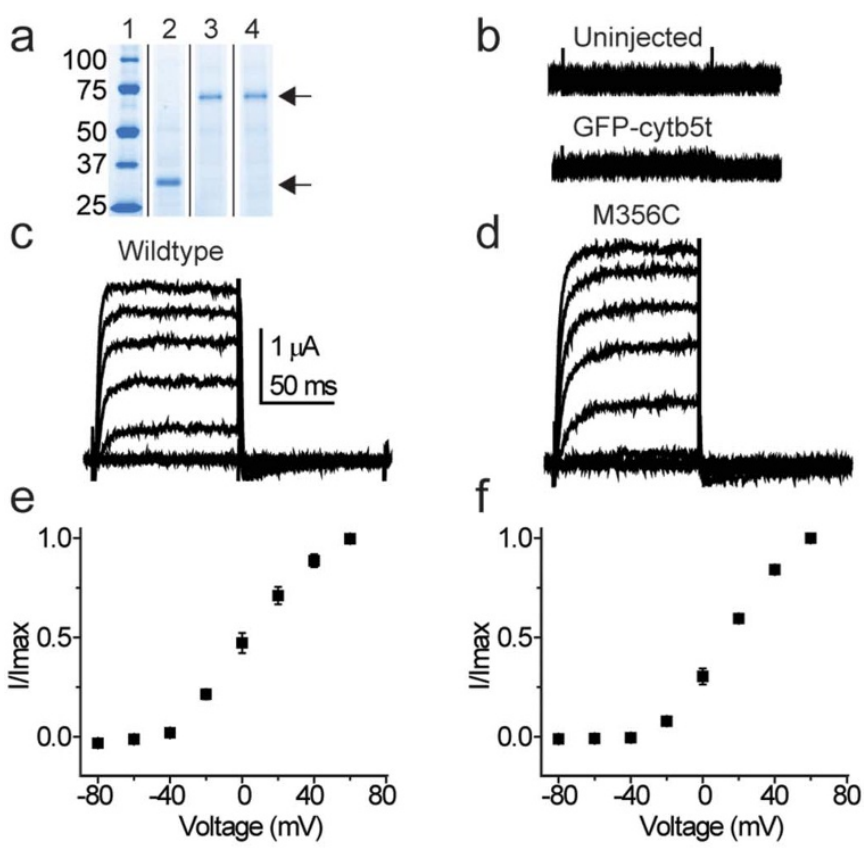

g

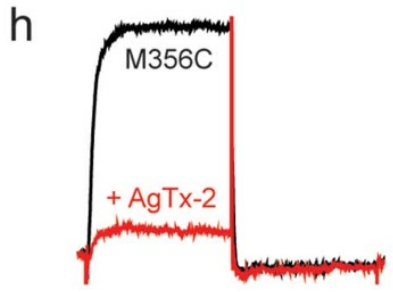

Figure $2 \mid$ Voltage-dependent response and pharmacology of injected samples produced from cell-free translation. (a) SDS-PAGE analysis of proteins produced by wheat-germ cell-free translation. Lanes 1 to 4 , representative gel slices from different experiments displaying, from left to right, the molecular weight marker (in $\mathrm{kDa}$ ), GFP-cytb5t (32 kDa), wildtype and M356C zh4IR channel monomers (71 kDa). The protein samples shown in the gel correspond to those used in other experiments shown in this figure. The family of current traces elicited in response to depolarizing voltage steps $(-80$ to $+60 \mathrm{mV}, \Delta 20 \mathrm{mV}$ ) for uninjected oocytes and oocytes injected with GFP-cytb5t (at $0.86 \mathrm{pmol}$ ) (b) were negligible compared to the wildtype channel (c) and the M356C channel (d) injected at 0.19 pmol. Current-voltage (I-V) relationship for wildtype (e) and mutant (f) Shaker channels. Inhibition of wildtype (g) and mutant (h) channels in the absence (black) and presence (red) of $10 \mathrm{nM}$ of the pore blocking compound AgTx-2. 
purification step is necessary for this approach. Application of agitoxin-2 (AgTx-2), a highly specific peptide toxin, reduced the outward currents by $>90 \%$ (Fig. $2 \mathrm{~g}$,h). These data indicate that channels in the correct orientation yield a majority of the outward currents, although we cannot rule out the possibility that some fraction of channels are inserted in the opposite 'inside out' orientation or silent ${ }^{20}$. Notably, orientation is an issue for ion channel reconstitution in artificial lipid bilayers, where functional incorporation efficiency is already low.

Determining the time and concentration dependence for functional studies in Xenopus oocytes. Next, we monitored the timecourse for appearance of voltage-dependent currents and the protein concentration-dependence of the response. For the timecourse experiments, wildtype channels were injected into oocytes and assayed over a $24 \mathrm{~h}$ period post-injection. Functional Shaker channels were produced within 3-4 h post-injection (Fig. 3a). Interestingly, we observed an increase in currents over $\sim 12 \mathrm{~h}$ time window, with the near doubling of $\mathrm{K}^{+}$currents suggesting a slow incorporation process. Next, we tracked individual oocytes from the same injection set over $24 \mathrm{~h}$ to determine the timepoint of maximal $\mathrm{K}^{+}$current. We observed a linear increase in currents $\left(\mathrm{R}^{2}=0.85\right)$ that reached a maximum after $\sim 12 \mathrm{~h}$ (Fig. $3 \mathrm{~b}$ ). Currents reduced $24 \mathrm{~h}$ after injection, which may signify a gradual increase in the protein turnover. This conclusion seems plausible because the proteincontaining vesicles are the limiting factor for incorporation and are not replenished after the initial injection. Interestingly, the timecourse for functional Shaker channels is similar to that reported for transplanted lipid vesicles containing nicotinic acetylcholine receptors from Torpedo electroplaques ${ }^{21}$.
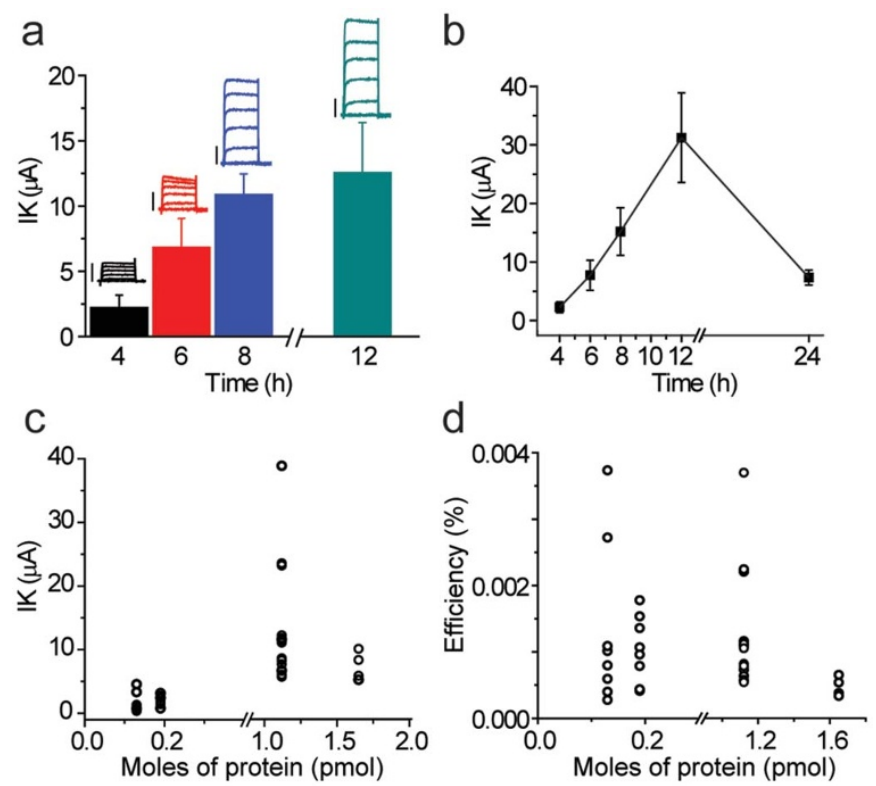

Figure 3 Timecourse and concentration-dependence of the appearance of zh4IR current in protein-injected samples. (a) Maximal $\mathrm{K}^{+}$current elicited at $+60 \mathrm{mV}$ averaged at varying times post-injection for wildtype ( $\Delta 4$ Cys) proteins. Representative families of currents (color-coded relative to the recording time) are displayed above the bars, with a $2 \mu \mathrm{A}$ scale bar. (b) Average timecourse of expression followed for the same oocyte over a period of 24 h post-injection $(n=4)$ with data points line-connected for trend comparison. (c) Comparison of maximal current elicited at $+60 \mathrm{mV}$ as a function of moles of tetrameric protein injected for four different injection sets. (d) Estimated incorporation efficiency (see Methods) for injection sets displayed in (c). Individual data points are shown in (c-d) to highlight the variability.
In an effort to estimate functional incorporation (Methods), the protein concentration-dependence of the response was examined. We observed a strong correlation $\left(\mathrm{R}^{2}=0.78\right)$ between the amount of protein injected and the $\mathrm{K}^{+}$current measured after $12 \mathrm{~h}$ (Fig. 3c). However, increasing the amount of protein injected beyond $1.2 \mathrm{pmol}$ per injection does not appear to increase current amplitude. While most oocytes tested yielded a similar response, in a few instances the currents were noticeably higher (Fig. 3c, 1.12 pmol protein). This is consistent with the observations of Miledi and co-workers ${ }^{11}$, who suggested outliers could be caused by variation in the number of channels injected into each oocyte from the viscous preparations and also by "clustered" insertion of the channels.

A useful parameter to evaluate the feasibility of this method in the context of other approaches is the functional incorporation efficiency. While we have shown that increasing the amount of protein injected strongly correlates to an increase in $\mathrm{K}^{+}$currents, not all of the Shaker channels may be functional, membrane-embedded, or in the 'correct' orientation. However, we can predict the number of channels required to yield a response at a given time and voltage $\left(\mathrm{N}_{\mathrm{obs}}\right)$ using the maximal current elicited at a given voltage, single channel conductance and reversal potential. This value $\left(\mathrm{N}_{\mathrm{obs}}\right)$ can be compared to the number of channels injected $\left(\mathrm{N}_{\mathrm{inj}}\right)$ using the concentration and molecular weight of the protein to estimate the efficiency of this system to incorporate functional Shaker channels into the oocyte membrane (Methods). Using these values, we observed an efficiency of $\sim 0.001-0.004 \%$ for functional channels incorporated into the oocyte membrane with a weak correlation $\left(\mathrm{R}^{2}=0.25\right)$ between the amount of protein injected and the efficiency (Fig. 3d). The functional incorporation efficiency of proteins synthesized from cell-free translation is strikingly similar to reconstituted channels from Torpedo electroplaques injected into oocytes ${ }^{20}$. It is important to note that this value does not take into consideration the orientation of the proteins incorporated or the fact that some proteins may be functionally silent or degraded upon injection. In theory, assuming an optimal system, one may be able to use these ratios to predict the amount of protein that needs to be injected to yield a specific current density. Furthermore, these estimates may be useful when preparing labeled samples fluorometric studies. While a relatively low number of synthesized channels are functionally incorporated into the oocyte membrane, the ability to manipulate the protein in vitro and transplant into a host system represent significant advantages for biophysical studies of eukaryotic IMPs.

Localization and uniformity of proteins within the oocyte membrane from proteoliposomes injections. To study the localization of the transplanted proteins into the oocyte plasma membrane, we utilized GFP-fused proteins (zh4IR-GFP and GFP-cytb5; Methods). Injection of mRNA (pBSTA vector) encoding the fluorescent zh4IR channel resulted in the appearance of GFP membrane fluorescence within 6-10 d using confocal microscopy (Fig. S4). Next, we assayed the proteoliposome samples containing the GFP-fused proteins. We compared the results from the GFP-fused potassium channel (zh4IRGFP) to a membrane anchored protein, GFP-cytb5t. GFP membrane fluorescence was readily detected for both GFP-fused proteins upon injection into the oocytes (Fig. 4a,b). This was in contrast to the lack of fluorescence detected from the mRNA-injected zh4IR-GFP and uninjected oocytes $12 \mathrm{~h}$ after injection (Fig. S4).

The localization and uniformity across the membrane was visually distinct between the two GFP-fused proteins from the proteoliposome injections (Supplementary Video). Membrane localization of zh4IR-GFP is in agreement with results from Le Caherec et al. ${ }^{16}$, who showed that injection of aquaporin-1 (AQP1) phosphatidylcholine proteoliposomes led to strong immunoreactivity of AQP1 in the plasma membrane, thus indicating efficient membrane incorporation of AQP1 over a similar timecourse ${ }^{16}$. Fluorescence from zh4IRGFP injected oocytes was primarily localized near the injection site 

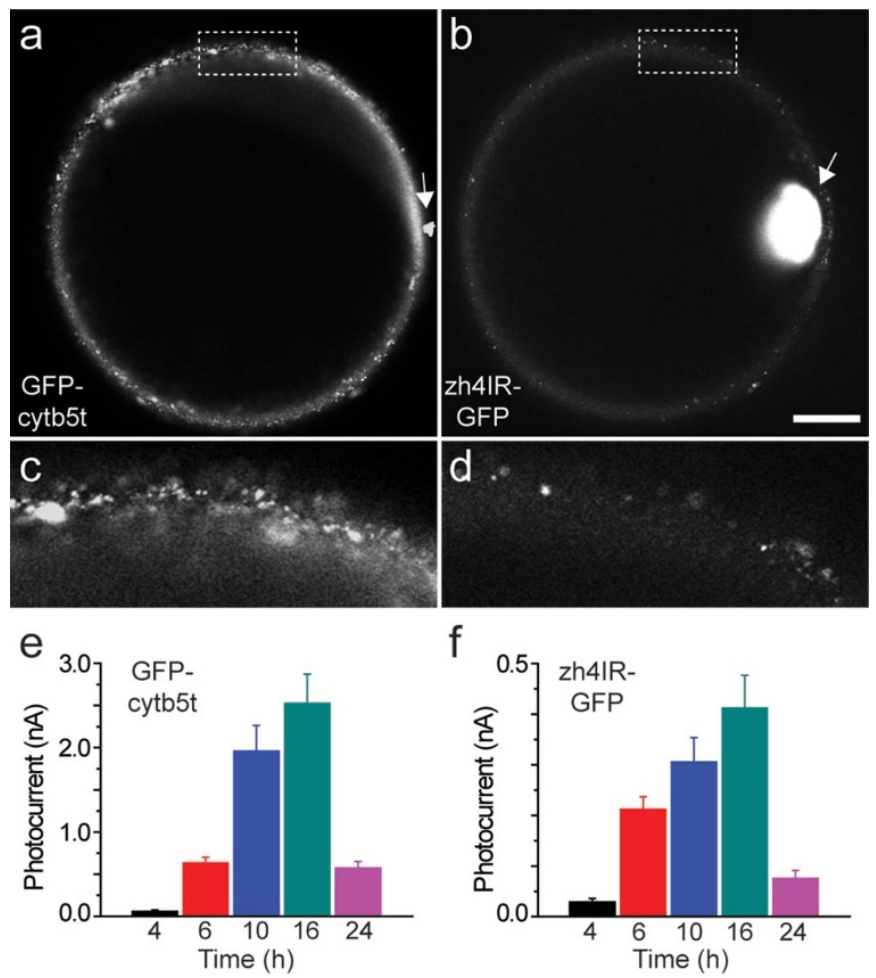

Figure $4 \mid$ Membrane localization of GFP fused proteins injected into Xenopus laevis oocytes. Grayscale confocal images of (a) GFP-cytb5t (at $2.77 \mathrm{pmol}$ ) and (b) zh4IR-GFP (at $0.58 \mathrm{pmol}$ ) protein localization within the oocyte membrane at $12 \mathrm{~h}$ after proteoliposome injections. Image intensity of the zh4IR-injected oocytes is increased 2-fold for ease of visualization. Arrows indicate the site of injection. Magnified membrane sections taken from the region within the dotted rectangle are displayed below. Scale bar $=200 \mu \mathrm{m}(\mathrm{a}, \mathrm{b})$ and $50 \mu \mathrm{m}$ (magnified regions, $\mathrm{c}, \mathrm{d}$ ). Corresponding membrane photocurrents (nA) were measured from GFPcytb5t (e) and zh4IR-GFP (f) at multiple times post-injection.

(Fig. 4b, arrow) suggesting a cluster of channels may be incorporated within that region, similar to that described by Morales et al. ${ }^{11}$ While GFP fluorescence was relatively faint throughout the rest of the membrane, noticeable puncta were also observed proximal to the injection site and diffusely spread throughout the membrane (Fig. 4b,d and Supplemental Video). In contrast, the signal detected for the GFP-cytb5t was spread throughout the entirety of the membrane with several discrete membrane puncta indicating efficient incorporation and lateral mobility within the lipid membrane (Fig. 4a,c).

Accordingly, photocurrents measured from the GFP-cytb5t were approximately 5-fold higher than oocytes injected with zh4IR-GFP (Fig. 4e,f). Differences observed between the two GFP-fused proteins may be due to the structural complexity (single pass vs. multipass homotetramer) of the fused proteins and lipid-protein interactions, thus influencing their ability to move laterally through the lipid membrane once incorporated (for review see ${ }^{22}$ ). In this case, it may be necessary to optimize lateral diffusion throughout the membrane by testing various lipid compositions and ratios for proteoliposome formation in combination with post-translational processing.

One potential complication in these experiments is that mRNA carried over from the in vitro translation reaction could be a major source of expressed channels in Xenopus oocytes. Although, we find that, when injected into oocytes, RNase A treated proteoliposomes (0.68 pmol) generate detectable currents (Fig S5), we further tested this possibility in two different ways. First, we generated mRNA from zh4IR-GFP in pEU-HSBC vector (background vector for in vitro translation) and then injected it into the oocytes. zh4IR-GFP
mRNA typically takes 7-8 d to express in contrast to injections of wildtype mRNA which results in current expression within 1-2 d. Using both confocal microscopy and our photodiode detection system, we observed no evidence of membrane expression within $24 \mathrm{~h}$ (Supplemental Table) suggesting that the mRNA derived from in vitro preparations was incapable of producing rapid expression which was characteristic of protein injections into the oocytes. Second, we fluorescently labeled the in vitro translated product with tetramethylrhodamine maleimide (TMRM). The maleimide group of the fluorophore predominantly reacts with free sulfhydryl groups, such as those found in cysteine residues, at neutral $\mathrm{pH}$. Upon labeling the synthesized proteins, we observed a noticeable shift in the SDSPAGE gel compared to the unlabeled protein (Fig. S6a) which indicates that the wildtype protein, without any engineered cysteines, was labeled in vitro. Wildtype channels contain seven cysteine residues per monomer, six of which (C96, C245, C301, C308, C462, C505) are located outside of the transmembrane segments ${ }^{23}$. Initial mass spectrometry of the labeled protein suggests that up to four cysteines per monomer were modified which results in $\sim 2 \mathrm{kDa}$ increase in mass. This is consistent with previous findings ${ }^{24,25}$ which show that extracellular (C245 and C462) and intracellular (C301 and C308) cysteines are accessible for chemical modification.

To determine whether the labeled proteins are functionally incorporated in oocyte membranes, we injected labeled proteoliposomes $(0.22 \mathrm{pmol}$ of proteins) into the oocytes. We observed an increase in membrane fluorescence at $6 \mathrm{~h}$ time point which nearly doubled by $12 \mathrm{~h}$ (Fig. S6c). The membrane fluorescence increased 18 fold in comparison to uninjected oocytes. Electrophysiological recordings at the $12 \mathrm{~h}$ time point showed that these channels were functional (Fig. S6b). Taken together, our findings provide strong evidence that the in vitro translated and labeled proteins were incorporated into oocyte membranes.

\section{Discussion}

The hybrid method described here provides a straightforward and efficient way to introduce membrane proteins synthesized by cellfree translation into a biological milieu. We chose a diverse set of membrane proteins (multipass IMPs, GFP fusions to the multipass protein, and a single pass membrane anchor) to test the feasibility and potential application of this approach.

We demonstrate that, in the cell-free mode, non-glycosylated Shaker potassium channels can be synthesized and incorporated into liposomes at quantities sufficient for rapid functional characterization in Xenopus oocytes. We show that channels produced in vitro display similar voltage-dependent properties and pharmacological sensitivity as mRNA injected samples. While we did not explicitly examine the mechanistic details of oligomerization in this communication, several groups have shown that proteins can be processed and oligomerize during cell-free preparation and functionally incorporate into artificial bilayers resembling proteins from a native membrane ${ }^{26-28}$. Additionally, at present we cannot state how our synthesized proteins incorporate into the oocyte membrane (constitutive or non-constitutive pathway) however, evidence from Gal and Morales points to a slow constitutive mechanism independent of intracellular calcium ${ }^{21}$. Additional experiments using our approach are needed to fully examine the method of channel incorporation. Our results, at a macroscopic level, are in agreement with single channel recordings obtained from Shaker potassium channels using cell-free methods to synthesize, process, and reconstitute into artificial bilayers ${ }^{26}$. While the incorporation appears low using this approach, it is not unreasonable compared to the current density observed for other proteoliposome injection studies using the oocyte host $^{15,20}$. Together, this strategy provides a versatile approach for protein synthesis, post-translational labeling, and functional studies of eukaryotic IMPs that may not be examined using current biochemical methods. 
There are several advantages of this approach for functional studies of modified ion channels when compared to current cell-based labeling techniques. First, our approach allows us to specifically label target proteins without any endogenous labeling which contributes to unnecessary background noise. Second, the extent of labeling can be precisely quantified which reduces uncertainties associated with modification studies. For instance, incomplete labeling in Förster resonance energy transfer (FRET) experiments can alter the FRET efficiencies and skew the measurements. In many instances, significant contamination of functional recordings by unmodified channels can complicate data interpretation. Third, our approach of in vitro modification can label sites that are inaccessible to cell-based labeling methods. At present, voltage-clamp fluorimetry measurements are restricted to sites accessible from the extracellular side. Therefore, our knowledge regarding the structural dynamics of sites that are either buried in the membrane or accessible from the cytosolic side remains limited. As such, we predict that our approach will greatly expand the scope of voltage-clamp fluorimetry studies. Fourth, our findings also suggest that the injected proteins bypass the normal cellular machinery and thus, this approach may be suitable for investigating proteins impaired in forward trafficking. For instance, the GFP-tagged Shaker mRNA typically takes a week to express, whereas when injected as proteoliposomes, photocurrents appear in oocyte membranes within few hours.

While our experiments described here demonstrate the feasibility of using this approach to functionally incorporate modified proteins into oocyte membranes, many questions remain outstanding. For instance, we do not know what fraction of the membrane-incorporated protein is inserted in 'correct' orientation. In our experiments, the lipid composition was unchanged and it will be important to determine whether varying the lipid composition and ratio increases the efficiency of incorporation. The precise lipid environment favoring optimal incorporation may differ based on the membrane protein of interest ${ }^{15,29}$. Increasing the functional incorporation efficiency will be the key to realize the full potential of the open configuration of cell-free system for studies involving proteins that are tagged by fluorescent probes or unnatural amino acids.

\section{Methods}

Plasmid preparation. Genes encoding the wildtype Shaker potassium channel (zh4IR), along with wildtype-“ $\Delta 4$ Cys" (C245V, C301S, C308S, C462A, T449F), S3S4 mutant (M356C), and zh4IR with GFP fused to the carboxy terminus (zh4IR-GFP) were subcloned from the pBSTA vector into a Flexi pEU-HSBC vector by introducing a $5^{\prime}-$ SgfI restriction site upstream of the start codon and a $3^{\prime}-P m e I$ at the stop codon by PCR, digesting the gene insert, and ligating into the parent vector ${ }^{33}$. pEU-HSBC can be obtained from the Protein Structure Initiative Materials Repository, http:// psimr.asu.edu. Site-directed mutagenesis was performed using the PCR-based QuikChange mutagenesis kit (Stratagene). Plasmid DNA from transformed E.coli cells (XL1 Blue) was harvested and purified using Qiagen mini and midi preparation kits and used for transcription reactions. The zh4IR-GFP (3304 bp; $97 \mathrm{kDa}$ monomer) fusion is a homotetrameric, non-conducting $(\Delta \mathrm{W} 434 \mathrm{~F})$ channel protein and retains, per subunit, the native six transmembrane segments and includes an eGFP (717 bp) fusion placed 13 residues downstream of the S6 segment at the carboxy terminus. A similar procedure was used to clone GFP-cytb5t into pEUHSBC. GFP-cytb5t consists of 37 residues from the carboxy terminus of human cytochrome b5 fused to GFP. The carboxy terminus has been shown to spontaneously insert into membranes and lipid bilayers ${ }^{30,31,27,28}$.

Preparation of samples for injection using cell-free translation. In vitro protein synthesis in the presence of liposomes was performed as reported ${ }^{3}$ with slight modifications. Briefly, the transcription reaction, which was typically done in a $100-\mu \mathrm{L}$ reaction or in a higher volume, consists of $0.1-0.2 \mathrm{mg} / \mathrm{mL}$ of proteinase $\mathrm{K}$ treated plasmid DNA, 4 mM NTPs, $1.6 \mathrm{U} / \mu \mathrm{L}$ SP6 RNA polymerase (Promega), and $0.8 \mathrm{U} / \mu \mathrm{L}$ RNase inhibitor (Promega), and $1 \times \mathrm{TB}+\mathrm{Mg}$ containing $80 \mathrm{mM}$ HEPES$\mathrm{KOH}$ (pH 7.8), $20 \mathrm{mM}$ magnesium acetate, $2 \mathrm{mM}$ spermidine hydrochloride, and $10 \mathrm{mM}$ dithiothreitol (DTT). Reactions were incubated at $37^{\circ} \mathrm{C}$ for $4 \mathrm{~h}$. Precipitated material was removed by centrifugation at $18000 \times g$ for $3 \mathrm{~min}$ at ambient temperature. The resultant supernatant was used for translation reaction or RNA purification. In an optimized procedure, RNA was further purified with RNeasy (Qiagen) or PureLink (Ambion) RNA purification kit. Typically about $5 \mathrm{mg} / \mathrm{mL}$ RNA solution was obtained after the purification. The translation reaction $(25 \mu \mathrm{L})$ consists of $6.25 \mu \mathrm{L}$ of WEPRO2240 or WEPRO2240H (wheat germ extract, CellFree Sciences), $5 \mu \mathrm{L}$ of RNA solution, $1.2 \mathrm{mg} / \mathrm{mL}$ soy liposomes, $0.3 \mathrm{mM} 20$ amino acids mixture, $0.04 \mathrm{mg} / \mathrm{mL}$ creatine kinase, and $1 \times \mathrm{DB}$ translation buffer containing $24 \mathrm{mM}$ HEPES-KOH (pH 7.8), $100 \mathrm{mM}$ potassium acetate, $2.5 \mathrm{mM}$ magnesium acetate, $0.4 \mathrm{mM}$ spermidine hydrochloride, $4 \mathrm{mM}$ DTT, $1.2 \mathrm{mM}$ ATP, $0.25 \mathrm{mM}$ GTP, $16 \mathrm{mM}$ creatine phosphate, and $0.005 \%(\mathrm{w} / \mathrm{v})$ sodium azide. The reaction was incubated at ambient temperature with dialyzing against $800 \mu \mathrm{L}$ of $1 \times \mathrm{DB}$ supplemented with $0.3 \mathrm{mM}$ amino acid mixture using a dialysis cup with 12,000 MWCO membranes (Lifetec Science Inc.). To extend the reaction time, $5 \mu \mathrm{L}$ of RNA solution was supplemented and the outer buffer was replenished every $24 \mathrm{~h}$.

Translation reactions were centrifuged at $18000 \times g$ for $6 \mathrm{~min}$ at ambient temperature to separate supernatant and pellet. The pellet was washed twice with suspending in a 0.6 volume of the translation reaction of a buffer containing $25 \mathrm{mM}$ HEPES- $\mathrm{NaOH}$ (pH 7.5), $100 \mathrm{mM} \mathrm{NaCl}$, and $2 \mathrm{mM}$ DTT and centrifuging. The washed pellet was suspended in the buffer of one-twentieth volume of the original translation reaction. Samples of cell-free synthesized proteins were used within $<24$ h of preparation.

RNase-treatment to reduce RNA carry-over was done by including $0.01 \mathrm{mg} / \mathrm{mL}$ RNase $\mathrm{A}$ in the first washing buffer and incubating for $10 \mathrm{~min}$ at ambient temperature before pelleting. The other procedures were done in the same as the standard preparation. The second washing buffer without RNase ensures to remove most of the RNase added.

SDS-PAGE and protein quantification. SDS-PAGE analysis was performed using Criterion stain-free Tris- $\mathrm{HCl} 4-20 \%$ polyacrylamide gels (BioRad) with Laemmli buffer system ${ }^{32,29}$. Gels were imaged by stain-free imager (Bio-rad), which utilizes a tryptophan-reactive agent to allow fluorescent quantification of protein bands in the gel. Synthesized protein concentrations were determined from SDS-PAGE band intensities as reported by the stain-free imager after comparison with protein standards (Precision Plus Protein Unstained Standards, Bio-rad). Software corrections take into account the tryptophan content of each protein. Whenever required, background band intensities were determined using control reactions that did not receive RNA addition, and subtracted from protein sample intensities. Gels were also imaged by Coomassie Brilliant Blue (CBB) staining and CCD photography.

Enzymatic deglycosylation. Samples from a zh4IR translation reaction were incubated at $80^{\circ} \mathrm{C}$ for $5 \mathrm{~min}$ in a buffer containing $0.5 \%(\mathrm{w} / \mathrm{v})$ SDS and $40 \mathrm{mM}$ DTT The denatured samples were diluted 2-fold into $50 \mathrm{mM}$ sodium phosphate ( $\mathrm{pH} 7.5)$, containing $1 \%(\mathrm{w} / \mathrm{v}) \mathrm{NP}-40$ and $50 \mathrm{U} / \mu \mathrm{L}$ PNGase F (New England Biolabs). The deglycosylation reaction was incubated at $37^{\circ} \mathrm{C}$ for $60 \mathrm{~min}$ and then analyzed by SDS PAGE.

TMRM labeling of synthesized proteins. Post-translational cysteine residue-specific labeling was performed in a suspension of pelleted protein using tetramethylrhodamine maleimide (TMRM; Invitrogen). Translation reactions were centrifuged at $18000 \times g$ for $3 \mathrm{~min}$ at ambient temperature to separate supernatant and pellet. In order to remove reducing agent before TMRM labeling, the pellet was washed twice with suspending in a 0.67 volume of the translation reaction of a buffer containing $25 \mathrm{mM}$ HEPES- $\mathrm{NaOH}$ (pH 7.5) and $100 \mathrm{mM} \mathrm{NaCl}$, and subsequent centrifuging. The washed pellet was labeled in the same way as the washing step except the buffer containing $50 \mu \mathrm{M}$ of TMRM and following incubation for $15 \mathrm{~min}$ at ambient temperature. The labeled pellet was washed twice with a buffer containing $25 \mathrm{mM}$ HEPES-NaOH (pH 7.5), $100 \mathrm{mM} \mathrm{NaCl}$, and $2 \mathrm{mM}$ DTT. The resultant pellet was suspended in one-twentieth volume of the original translation reaction.

Oocyte injection, electrophysiology and analysis. Xenopus laevis oocytes were chosen as an expression host for these studies because their size allows for precise delivery of large amounts of protein via microinjection, thus ensuring reproducibility. Oocytes (stage IV or V) were surgically removed in a manner consistent with the guidelines of the Animal Care and Use Committee at the University of WisconsinMadison and stored in $5 \mathrm{mM}$ HEPES, pH 7.6 adjusted with $\mathrm{NaOH}$, containing $96 \mathrm{mM} \mathrm{NaCl}, 2 \mathrm{mM} \mathrm{KCl}, 1.8 \mathrm{mM} \mathrm{CaCl}_{2}, 1 \mathrm{mM} \mathrm{MgCl}_{2}, 100 \mathrm{mg} / \mathrm{mL}$ of bovine serum albumin, and $100 \mu \mathrm{g} / \mathrm{mL}$ of gentamicin. Oocytes were stored on ice prior to injection. Selected oocytes were placed into an injection chamber filled with the above buffer lacking calcium. Oocytes were microinjected near the vegetal pole equator with $50 \mathrm{~nL}$ of proteoliposomes at the specified concentration.

A range of concentrations of reconstituted protein (assuming tetramer; $\sim 0.13$ to $1.65 \mathrm{pmol}$ ) were injected in these studies. As an additional control, the unpurified translation mixture was injected into oocytes. Injection of the unpurified translation mixture severely compromised the oocyte health and rendered oocytes inadequate for further experiments. To facilitate injections, the diameter of the microinjection electrode was carefully fashioned to accommodate the sample concentration and viscosity. We found the size of the tip was critical for obtaining consistent and repeatable injections while maintaining as small of a wound as possible upon oocyte injection. Thus the tip diameter ranged from $5-10 \mu \mathrm{m}(<1 \mathrm{pmol}$ protein) to $15-$ $25 \mu \mathrm{m}(>1$ pmol protein). Three to four hours were required after the initial protein injection for the oocyte to fully recover from the injection procedure and to allow reliable detection of outward $\mathrm{K}^{+}$current.

Injected oocytes were incubated at $18^{\circ} \mathrm{C}$ in $5 \mathrm{mM}$ HEPES, pH 7.6 adjusted with $\mathrm{NaOH}$, containing $96 \mathrm{mM} \mathrm{NaCl}, 2 \mathrm{mM} \mathrm{KCl}, 1.8 \mathrm{mM} \mathrm{CaCl}_{2}, 1 \mathrm{mM} \mathrm{MgCl}_{2}, 100 \mu \mathrm{g} /$ $\mathrm{mL}$ gentamicin, $50 \mathrm{U} / \mathrm{mL}$ penicillin and $50 \mu \mathrm{g} / \mathrm{mL}$ streptomycin. The oocyte incubation solution was exchanged every hour after injection. Recording electrodes were always placed within the animal pole for current measurements. Ionic current recordings were obtained at room temperature (4-24 h post-injection) using a 
two-electrode voltage-clamp setup (OC-725C Oocyte Clamp; Warner Instruments) with an external solution composed of $10 \mathrm{mM}$ HEPES, $\mathrm{pH}$ adjusted with 7.4 with 2morpholinoethanesulfonic acid, and containing $100 \mathrm{mM} \mathrm{NaOH}, 5 \mathrm{mM} \mathrm{KOH}, 2 \mathrm{mM}$ $\mathrm{Ca}(\mathrm{OH})_{2}$. The electrodes had a resistance less than $1 \mathrm{M} \Omega$. Analog signals were sampled at $20 \mathrm{kHz}$ with a Digidata $1320 \mathrm{~A}$ and filtered at $1 \mathrm{kHz}$. Linear leak was subtracted online using a $\mathrm{P} / 4$ protocol with a sub-pulse holding level of $-120 \mathrm{mV}$. Oocyte holding potential was $-80 \mathrm{mV}$ for all ionic current recordings. Photocurrent measurements, an indicator of membrane fluorescence, were obtained using a photodiode detection system equipped with a Uniblitz shutter (VCMD1, Vincent Associates) mounted onto the two-electrode voltage-clamp setup. Oocytes were positioned using an Olympus BX51W1 upright microscope using a PlanN $4 \times / 0.10$ objective. Membrane fluorescence was detected by focusing a LumplanFl $40 \times / 0.80$ water immersion objective onto the animal pole of an oocyte. Light $(84 \mathrm{~W}, 2.36 \mu \mathrm{A})$ from a xenon lamp source (Newport Corp.) was filtered with a 480/HQ40 bandpass filter and split using a 505LP dichroic mirror (Chroma Technology Corp.). Emitted light was filtered with a 535/HQ50 bandpass filter and focused onto a PIN-020A photodiode (UDT Instruments) by a condenser lens. The photodiode was connected to the integrating headstage of a patch clamp amplifier (EPC7, HEKA Elektronik). All data were acquired using Clampex (Molecular Devices) and analyzed with Clampfit, Excel (Microsoft), or Origin (GE Healthcare). Statistical analyses for small sample sizes using a two-sample unpaired t-test were performed using Origin where $\mathrm{p}<0.05$ was accepted as statistically significant. Graphical data are presented as average \pm s.e.m. and ' $n$ ' represents the number of samples. Images were constructed using Adobe Photoshop or Illustrator from the Creative Suites 5 software package (Adobe Systems Inc.).

Estimation of functional incorporation efficiency. The following equation was used to determine the number of channels incorporated:

$$
\text { Nobs }=\frac{I}{r(V-\text { Erev })}
$$

To estimate the relative number of channels incorporated the peak steady-state $\mathrm{K}^{+}$ current $(I)$ observed at $(V)+60 \mathrm{mV}$ for the injected oocytes at a particular postinjection time point, single channel conductance $(\gamma)$ for Shaker channels ${ }^{33}$ (assuming a single channel current of $\sim 2 \mathrm{pA}$ using a similar concentration gradient), and a reversal potential (Erev) estimated around $-65 \mathrm{mV}$ from tail currents obtained during hyperpolarizing steps from a prepulse at $+60 \mathrm{mV}$ were used. We defined the product as the number of observed channels $\left(\mathrm{N}_{\mathrm{obs}}\right)$. We then approximated the number of channels injected by determining the amount of protein injected (pmol protein $/ 50 \mathrm{~nL}$ volume) per oocyte using the mass of protein injected and dividing by the molecular weight for the non-glycosylated tetramer $(285 \mathrm{kDa})$. This value yielded the number of injected channels $\left(\mathrm{N}_{\mathrm{inj}}\right)$. The ratio of $\mathrm{N}_{\mathrm{obs}} / \mathrm{N}_{\mathrm{inj}}$ was used to determine the incorporation efficiency $(\mathrm{E})$ for the proteoliposome injections.

Confocal microscopy. Images were acquired using a Nikon A1 laser scanning confocal microscope (Nikon Instruments Inc.) with an inverted Nikon Eclipse Ti-E as the base microscope. Healthy oocytes ( $\sim 1 \mathrm{~mm}$ diameter) stored in six well plates in the same incubation solution described above were used for imaging. Oocytes selected for confocal imaging had uniform animal and vegetal poles. All imaging was performed at room temperature using a $488 \mathrm{~nm}$ laser for GFP excitation and a PlanFluor $10 \times$ objective with a working distance of $16.0 \mathrm{~mm}$ and a numerical aperture of 0.30 . Fluorescence emission was detected using a photomultiplier tube (PMT) following a 525/50 nm band-pass filter. The pinhole radius was set to $4.5 \mathrm{AU}$. Volume scans (z-series) were collected for analysis stepping through in $25 \mu \mathrm{m}$ increments. Confocal images are displayed at a z-series depth of $\sim 372 \mu \mathrm{m}$ from the near outer surface of the oocyte. The pinhole diameter, offset, PMT gain, and laser power were identical for all samples. For video rendering each 360 degree rotation was generated by producing a maximum intensity projection every 3.6 degrees using a series of confocal XY-images collected every $24.82 \mu \mathrm{m}$ through the depth of the oocyte. All image collection, three-dimensional projections and rotations, and measurements were performed using the NIS-Elements software (Nikon Inc.)

1. Tapper, A. R. \& George, A. L. Jr. Heterologous expression of ion channels. Methods Mol Biol 217, 285-294 (2003).

2. Miller, C. Ion channel reconstitution. (Plenum Press, New York; 1986).

3. Goren, M. A., Nozawa, A., Makino, S., Wrobel, R. L. \& Fox, B. G. Cell-free translation of integral membrane proteins into unilamelar liposomes. Methods Enzymol 463, 647-673 (2009).

4. Goren, M. A. \& Fox, B. G. Wheat germ cell-free translation, purification, and assembly of a functional human stearoyl-CoA desaturase complex. Protein Expr Purif 62, 171-178 (2008).

5. Sevova, E. S. et al. Cell-free synthesis and functional characterization of sphingolipid synthases from parasitic trypanosomatid protozoa. J Biol Chem $\mathbf{2 8 5}$, 20580-20587 (2010)

6. Sonar, S., Patel, N., Fischer, W. \& Rothschild, K. J. Cell-free synthesis, functional refolding, and spectroscopic characterization of bacteriorhodopsin, an integral membrane protein. Biochemistry 32, 13777-13781 (1993).

7. Nozawa, A. et al. A cell-free translation and proteoliposome reconstitution system for functional analysis of plant solute transporters. Plant Cell Physiol 48, $1815-1820$ (2007).
8. Barnard, E. A., Miledi, R. \& Sumikawa, K. Translation of exogenous messenger RNA coding for nicotinic acetylcholine receptors produces functional receptors in Xenopus oocytes. Proc R Soc Lond B Biol Sci 215, 241-246 (1982).

9. Selenko, P., Serber, Z., Gadea, B., Ruderman, J. \& Wagner, G. Quantitative NMR analysis of the protein $\mathrm{G} \mathrm{B1}$ domain in Xenopus laevis egg extracts and intact oocytes. Proc Natl Acad Sci U S A 103, 11904-11909 (2006).

10. Marsal, J., Tigyi, G. \& Miledi, R. Incorporation of acetylcholine receptors and Clchannels in Xenopus oocytes injected with Torpedo electroplaque membranes. Proc Natl Acad Sci U S A 92, 5224-5228 (1995).

11. Morales, A. et al. Incorporation of reconstituted acetylcholine receptors from Torpedo into the Xenopus oocyte membrane. Proc Natl Acad Sci U S A 92, 8468-8472 (1995).

12. Palma, E. et al. Microtransplantation of membranes from cultured cells to Xenopus oocytes: a method to study neurotransmitter receptors embedded in native lipids. Proc Natl Acad Sci U S A 100, 2896-2900 (2003).

13. Palma, E. et al. Physiological characterization of human muscle acetylcholine receptors from ALS patients. Proc Natl Acad Sci U S A 108, 20184-20188 (2011),

14. Miledi, R., Eusebi, F., Martinez-Torres, A., Palma, E. \& Trettel, F. Expression of functional neurotransmitter receptors in Xenopus oocytes after injection of human brain membranes. Proc Natl Acad Sci U S A 99, 13238-13242 (2002).

15. Schmidt, D. \& MacKinnon, R. Voltage-dependent $\mathrm{K}+$ channel gating and voltage sensor toxin sensitivity depend on the mechanical state of the lipid membrane. Proc Natl Acad Sci U S A 105, 19276-19281 (2008).

16. Le Caherec, F. et al. Incorporation of proteins into (Xenopus) oocytes by proteoliposome microinjection: functional characterization of a novel aquaporin J Cell Sci 109 (Pt 6), 1285-1295 (1996).

17. Junge, F. et al. Large-scale production of functional membrane proteins. Cell Mol Life Sci 65, 1729-1755 (2008)

18. Santacruz-Toloza, L., Huang, Y., John, S. A. \& Papazian, D. M. Glycosylation of shaker potassium channel protein in insect cell culture and in Xenopus oocytes. Biochemistry 33, 5607-5613 (1994).

19. Schmidt, D., Jiang, Q. X. \& MacKinnon, R. Phospholipids and the origin of cationic gating charges in voltage sensors. Nature 444, 775-779 (2006).

20. Ivorra, I. et al. Protein orientation affects the efficiency of functional protein transplantation into the xenopus oocyte membrane. J Membr Biol 185, 117-127 (2002).

21. Gal, B., Ivorra, I. \& Morales, A. Functional incorporation of exogenous proteins into the Xenopus oocyte membrane does not depend on intracellular calcium increase. Pflugers Arch 440, 852-857 (2000).

22. Jacobson, K., Ishihara, A. \& Inman, R. Lateral diffusion of proteins in membranes. Annu Rev Physiol 49, 163-175 (1987).

23. Boland, L. M., Jurman, M. E. \& Yellen, G. Cysteines in the Shaker K+ channel are not essential for channel activity or zinc modulation. Biophys J 66, 694-699 (1994).

24. Mannuzzu, L. M., Moronne, M. M. \& Isacoff, E. Y. Direct physical measure of conformational rearrangement underlying potassium channel gating. Science 271, 213-216 (1996).

25. Holmgren, M., Jurman, M. E. \& Yellen, G. N-type inactivation and the S4-S5 region of the Shaker K+ channel. J Gen Physiol 108, 195-206 (1996).

26. Rosenberg, R. L. \& East, J. E. Cell-free expression of functional Shaker potassium channels. Nature 360, 166-169 (1992).

27. Syeda, R., Santos, J. S., Montal, M. \& Bayley, H. Tetrameric assembly of KvLm K+ channels with defined numbers of voltage sensors. Proc Natl Acad Sci U S A 109, 16917-16922 (2012).

28. Falk, M. M., Buehler, L. K., Kumar, N. M. \& Gilula, N. B. Cell-free synthesis and assembly of connexins into functional gap junction membrane channels. EMBO J 16, 2703-2716 (1997)

29. Fernandez-Carvajal, A. M. et al. Structural and functional changes induced in the nicotinic acetylcholine receptor by membrane phospholipids. J Mol Neurosci 30, 121-124 (2006)

30. Ozols, J. Structure of cytochrome b5 and its topology in the microsomal membrane. Biochim Biophys Acta 997, 121-130 (1989).

31. Hanlon, M. R., Begum, R. R., Newbold, R. J., Whitford, D. \& Wallace, B. A. In vitro membrane-inserted conformation of the cytochrome b(5) tail. Biochem J352 Pt 1, $117-124$ (2000)

32. Laemmli, U. K. Cleavage of structural proteins during the assembly of the head of bacteriophage T4. Nature 227, 680-685 (1970).

33. Yellen, G., Jurman, M. E., Abramson, T. \& MacKinnon, R. Mutations affecting internal TEA blockade identify the probable pore-forming region of a $\mathrm{K}+$ channel. Science 251, 939-942 (1991).

\section{Acknowledgements}

We thank members of the University of Wisconsin-Madison Center for Eukaryotic Structural Genomics (CESG) their valuable comments and Sandipan Chowdhury for his extremely helpful and insightful discussions. We also greatly appreciate assistance from Lance Rodenkirch and Michael Hendrickson of the W.M. Keck Laboratory for Biological Imaging for their aid with the confocal images and analysis. This work was funded by National Institutes of Health grants 5T32HL007936-09 to B.W.J., GM084140 to B.C. and U54 GM094584-01 to B.G.F. 


\section{Author contributions}

B.W.J. carried out all oocyte microinjections, molecular biology, electrophysiology, fluorescence and confocal experiments. S.M. and E.T.B. conducted the cell-free experiments. B.W.J., S.M., B.G.F. and B.C. contributed to the experimental design and manuscript preparation.

\section{Additional information}

Supplementary information accompanies this paper at http://www.nature.com/ scientificreports
Competing financial interests: The authors declare no competing financial interests.

License: This work is licensed under a Creative Commons

Attribution-NonCommercial-NoDerivs 3.0 Unported License. To view a copy of this license, visit http://creativecommons.org/licenses/by-nc-nd/3.0/

How to cite this article: Jarecki, B.W., Makino, S., Beebe, E.T., Fox, B.G. \& Chanda, B. Function of Shaker potassium channels produced by cell-free translation upon injection into Xenopus oocytes. Sci. Rep. 3, 1040; DOI:10.1038/srep01040 (2013). 